\title{
Participación juvenil en la construcción de la blogosfera
}

\section{Youth participation in building the blogosphere}

Patricia González Aldea, Universidad Carlos III de Madrid - patricia.gonzalez.aldea@uc3m.es

Carme Marta Lazo, Universidad de Zaragoza - cmarta@unizar.es

Marta Redondo García, Universidad de Valladolid - marta.redondo@hmca.uva.es

Resumen

Esta comunicación parte de los resultados de una investigación del Observatorio del Ocio y Entretenimiento Digital (OCENDI) que analiza las principales tendencias de consumo de blogs entre los jóvenes universitarios españoles (18-25 años).

Según dichos resultados, la participación en la blogosfera se reduce a la lectura en muchos casos, siendo limitada la interacción a través de comentarios y algo más activa en redes sociales. La influencia y poder prescriptor de la blogosfera en estos jóvenes, -y a pesar de la credibilidad que dan a los bloggers-, se muestra también en declive atendiendo al consumo hiper-fragmentado de blogs, la escasa fidelización y a sus opiniones sobre la saturación de la blogosfera..

Palabras clave

Blogs, jóvenes, participación, blogosfera.

Abstract

This communication is based on the results of a study by the Observatorio del Ocio y Entretenimiento Digital (OCENDI) that analyses the main trends in blog consumption among young Spanish university students (18-25 years old).

According to these results, participation in the blogosphere is reduced to reading in many cases, with limited interaction through comments and somewhat more active in social networks. The influence and prescriptive power of the blogosphere in these young people, and despite the credibility they give to bloggers, is also in decline due to the hyper-fragmented consumption of blogs, low loyalty and their opinions on the saturation of the blogosphere.

Palabras clave

Blogs, jóvenes, participación, blogosfera. 


\section{Introducción}

En 1997, el escritor norteamericano Jorn Barger acuña el término weblog que traduce como "logging the web" o haciendo un diario de la web. En esa etapa inicial los blogs eran una herramienta muy limitada, utilizada por pocos internautas que empleaban sus blogs principalmente para compartir enlaces interesantes.

Sin embargo, la aparición de gestores gratuitos de contenidos, entre ellos: Blogger, Blogspot, Wordpress, Bloggia o Tumblr, técnicamente sencillos y por tanto al alcance de una mayoría de internautas, provoca que los blogs vivan una etapa de expansión que coincide con la aparición de numerosos estudios que saludan el fenómeno como la posibilidad de generar un pensamiento crítico y alternativo al oficial.

Antúnez escribía en 2005 que el blog estaba llamado a ser "protagonista de una revolución comparable a la que provocó la aparición de la imprenta en el siglo XV" (2005, p. 51) propiciando la verdadera "democratización en el proceso comunicativo". Para Arroyas (2006), la blogosfera iba a ofrecer a la comunicación y al periodismo "una conexión con ese mundo de la vida independiente del poder económico y político" aportando al debate público los asuntos que realmente preocupaban al ciudadano y no a la élite. En su opinión, sería una vía para llevar a la agenda la interpretación del mundo de los ciudadanos sirviendo a la redefinición del espacio público.

Si 2005 supuso el momento de explosión de las bitácoras en el ámbito hispano (Tricas et al., 2006), en estos momentos el fenómeno vive una etapa de retraimiento. Aunque el número de blogs abiertos en España es muy elevado -se estima entre 864.000 y 1.890 .000 según las diversas fuentes (Bitácoras.com, 2012)- sólo un $28 \%$ de los blogueros reconoce que lo utiliza y un $7 \%$ que lo actualiza con frecuencia (Fundación Orange, 2012, p.144).

Islas (2010) entiende esta fase como un periodo de ajuste del fenómeno provocado, en buena medida, por la extensión de las redes sociales: espacialmente Twitter considerada desde sus inicios como un sistema de microblogging. Se explicaría como un movimiento de selección natural de especies digitales, de modo que algunas de las funciones que se desarrollaban a través de blogs han migrado a redes sociales que las cumplen de manera más eficaz. De hecho, el informe 2013 del Observatorio Nacional de las Telecomunicaciones y de la Sociedad de la Información señala que crear websites o blogs se sitúa a la cola en cuanto a los usos de internet que hacen los ciudadanos españoles con un $9 \%$, muy lejos de los porcentajes de las primeras actividades: envío de mensajes, realizado por el $62 \%$ de los usuarios y búsqueda de información sobre bienes y servicios por el $61 \%$.

\section{Jóvenes como autores de blogs}

El bloguero hispano, tal y como lo recogen Garrido y Lara (2008), es joven (el $51 \%$ tiene entre 20 y 30 años), con estudios universitarios (70\%), de sexo masculino (73\%) y con experiencia en la Red (un $76 \%$ de más de 5 años). Esa juventud de los miembros de la bloggosfera resulta previsible dada la mayor competencia tecnológica de los que Prensky denominó en 2001 "nativos digitales", que han crecido acompañados de internet y haciendo uso de múltiples pantallas donde obtener información, diversión y a través de las cuales interactuar con otros. Es la denominada "i-Generación o generación interactiva que ha convivido desde su nacimiento con normalidad en un mundo en el que las pantallas forman parte de su hábitat de referencia diaria" (Marta-Lazo, Martínez-Rodrigo y Sánchez-Martín, 2012: 42).

Los jóvenes y adolescentes tienen acceso a numerosas tecnologías digitales tanto en el ámbito doméstico como a través de los dispositivos móviles. De modo que el proceso natural es la apropiación por parte de este sector de las diversas herramientas disponibles para hacer frente a sus necesidades y aficiones cotidianas. Como señalan Aranda, Sánchez et al. (2010) refiriéndose a los adolescentes:

Su vida se desarrolla en contextos caracterizados por la creciente presencia de medios y tecnología, y en los que las tecnologías digitales juegan un papel fundamental en relación con múltiples aspectos de su vida cotidiana, como la sociabilidad, el consumo o el aprendizaje.

Las características propias de los blogs los hacen proclives a la cultura juvenil dado sus mensajes breves, inmediatos, multimediáticos y multitemáticos. Pero también debido a la necesidad psicológica -que caracteriza a la juventud- de generar un espacio propio dentro de la sociedad, un lugar donde hace oír a los demás la voz individual.

Para Martínez Sánchez (2008), el blog sirve para construir la propia identidad, es "una herramienta de la personalidad, una necesidad de dar forma a lo que uno mismo es o quiere mostrar a los ojos y mente de los otros". Por eso cumplen entre la población joven la misma función que la escritura de unas memorias digitales:

Los bloggers asumen a su blog como un espacio personal, propio, algo construido por y para el propio autor, en el presente y para el futuro, a manera de memoria o rastro de lo que se ha sido $y$ cómo se ha llegado a ser lo que se es" (Flores, 2010).

Los blogs como fruto personal de su autor son temática y estéticamente muy diversos aunque su materia prima principal son noticias, comentarios y opiniones que nacen para ser compartidas con otros, con quienes además se pueden establecer conversaciones y debates. Por lo tanto formalmente, aunque no en tan buena medida como las redes sociales, servirían para estar en contacto con un 


\section{La blogosfera, una herramienta de información y de expresión personal}

La oferta existente en la blogosfera es enormemente variada tanto en la temática como en los objetivos de los autores de las bitácoras. Entre las funciones que cumplen los blogs, Leibrandt (2006) señala las siguientes: transmitir novedades, abrir debates, intercambiar información y conocimientos, acopiar e intercambiar enlaces, establecer y mantener contactos personales o presentar trabajos y artículos propios. Por tanto, la blogosfera se constituye como una red dinámica capaz de satisfacer buena parte de las necesidades comunicativas de los jóvenes. Es además un "espacio extenso sin límites definidos" (Estalella, 2005) que no se circunscribe a contactos establecidos previamente sino que está abierto a la consulta y participación de cualquier internauta.

Pero el blog sirve además como expresión personal del autor y contribuye a "la construcción de la identidad individual" (Estalella, 2005) y a generar un sentimiento de pertenencia a la comunidad, un elemento a tener en cuenta dado que la etapa de la juventud se caracteriza por una búsqueda de referencias externas que se encuentran en la familia, los amigos, los medios de comunicación pero, evidentemente y de manera creciente en Internet y en los múltiples contactos que se establecen a través de la red.

Se confirma además que son los jóvenes quienes con más frecuencia y durante más tiempo hacen uso de la red. El estudio Retratos de Juventud del Observatorio Vasco de la Juventud (2012) confirma que el $96 \%$ de los jóvenes usa Internet y que la mayoría (81\%) lo hace a diario. Además, el $53 \%$ de los jóvenes considera que Internet es el medio ideal para entretenerse (frente al $16 \%$ de los adultos) y el $66 \%$ de los jóvenes de entre 15 y 29 años optaría por internet si sólo pudiese tener acceso a un medio de comunicación.

Por todas las funcionalidades que cumple la blogosfera, habría que deducir que la participación de los jóvenes en ella es alta, tanto a la hora de crear sus propias bitácoras que sirvan de vía de expresión personal, como a la de interactuar con los autores de otros blogs.

\section{Poder y prescripción en la blogosfera}

El poder o influencia de estas publicaciones en Internet es muy dispar. En ocasiones, tiene relación inequívoca con la temática, como es el caso de las páginas dedicadas a la tecnología, la información de actualidad o el fútbol; en otras, la relación de importancia se establece con la firma conocida que escribe, como es el caso de políticos y periodistas de prestigio. Sin embargo, hay que tener presente que la citada influencia es muy cambiante y que no es lo mismo medir el poder de un blog independiente que el de uno profesional. Detrás de una página independiente, el blogger es el creativo ,suele estar solo, y utiliza el blog como hobby, con lo que la distribución es reducida y lenta; en el caso de un blog profesional, hay todo un equipo de infraestructuras de difusión y distribución complejo que consigue que los mensajes publicados alcancen a más público rápidamente.

Para medir el poder de prescripción que ejercen los blogs sobre el público se tienen en cuenta multitud de parámetros, entre los que destacan, los usuarios únicos, las visitas, el número de seguidores de la página en redes sociales, la red de enlaces al blog que aparecen en otros blogs distintos o el share de sus artículos en las redes sociales, Facebook y Twitter, especialmente. También se observa las veces que sus entradas son referenciadas en otros medios de difusión fuera de Internet aludiendo a ellos como "medios especializados" en alguna materia.

Según datos ofrecidos en marzo de 2013 por Ebuzzing Social el ranking de blogs está encabezado por ALT1040 (http://alt1040.com), con 183.074 seguidores en Twitter y más de 50.000 en Facebook, se presenta como una guía del geek (amante de la tecnología y la informática) con noticias y opiniones de internet, cultura digital, tecnología y propiedad intelectual. En el top que publica este laboratorio social, sobre el análisis de más de 30 categorías temáticas diferentes de weblogs españoles, figuran al menos otros cinco blogs sobre tecnología -MuyLinux, Microsiervos, Applesfera o Xataka-, uno sobre arte y cultura, Yorokobu, y otro sobre gastronomía, El Comidista, del diario El País.

Ante este panorama, sería plausible afirmar que los intereses más cercanos a los jóvenes de hoy se encontrarían en los blogs dedicados a aspectos como la tecnología, los medios de comunicación, los deportes o los videojuegos, aunque no es fácil encontrar estudios que abarquen este asunto. Resulta relevante, en consecuencia, efectuar un primer acercamiento a esta realidad para determinar cuáles son sus páginas preferidas y sobre qué temas construyen ellos sus blogs particulares con el objetivo de averiguar hasta qué punto es una herramienta social para este colectivo.

\section{Metodología}

El objetivo de esta comunicación es analizar la tendencia de participación en blogs de los jóvenes españoles universitarios con edades comprendidas entre los 18 y los 25 años.

La hipótesis de partida es que los blogs han perdido poder prescriptor dentro de este colectivo en favor del acelerado uso de las redes sociales. En este contexto, tenemos que aunque los jóvenes españoles acceden a los blogs de forma habitual, muchos a diario, son una minoría aquellos que cuentan con un blog propio, así como los que participan de un modo activo en ese universo, en lo 
Este estudio está basado en la realización de 500 encuestas aleatorias a jóvenes de entre 18 y

25 años, observando las variables de género, edad, estudios (universitarios y relacionados con la comunicación) y población, con medias ponderadas por regiones (según cifras del INE de 1 de enero de 2012), de forma que queden representadas las actitudes y preferencias de los jóvenes españoles de todas las Comunidades Autónomas que constituyen el país.

Tabla 1: Distribución proporcional de la muestra por Comunidades Autónomas

\begin{tabular}{|l|l|}
\hline Comunidad Autónoma & Entrevistas \\
\hline Andalucía & 89 \\
\hline Cataluña & 82 \\
\hline Madrid & 72 \\
\hline Valencia & 54 \\
\hline Galicia & 30 \\
\hline Castilla y León & 27 \\
\hline País Vasco & 23 \\
\hline Canarias & 23 \\
\hline Castilla La Mancha & 22 \\
\hline Murcia & 16 \\
\hline Aragón & 14 \\
\hline Asturias & 11 \\
\hline Extremadura & 11 \\
\hline
\end{tabular}

Fuente: elaboración propia

El muestreo cuantitativo basado en los cuestionarios para la recogida de información es, por tanto, un muestreo por cuotas no probabilístico e intencionado, que representa una muestra significativa para iniciar un acercamiento al objeto de estudio que derive en un primer diagnóstico sobre la situación actual de cambio en el consumo de blogs que planteamos en las hipótesis.

Se les han formulado 14 preguntas cerradas y 2 abiertas encaminadas a confirmar sus hábitos de consumo respecto de los blogs, además de conocer su opinión sobre la oferta actual.

\section{Resultados}

Poder prescriptor en la blogosfera juvenil

En los resultados de nuestro estudio, las preferencias de los jóvenes, con excepción de la política, apuntan a una mayoría de temas que corresponden a la que se podría categorizar como "información práctica y de entretenimiento": cine, moda, música y deportes.

Sobre el poder prescriptor e influencia de los blogs en la blogosfera juvenil, los datos muestran un consumo hiper-fragmentado donde la elección y mención de blogs llega a ser tan diversa y amplia como los gustos personales de cada lector de blogs. Esto se pudo comprobar en los resultados de este estudio en la pregunta abierta en la que se les solicitaba el nombre de sus dos blogs preferidos. De los 500 encuestados se obtuvo sólo una lista de 438 blogs como respuesta, es decir, menos de la mitad de la cantidad a obtener si todos hubiesen respondido a la pregunta. Este dato confirma también que los blogs no son hoy ni tan leídos ni tan seguidos por los jóvenes como apuntaba la tendencia general de consumo de blogs iniciada la década pasada con lo que, a su vez, pone de relieve el hecho de que sea cada vez más complicado tener poder e influencia en medio de una blogosfera muy saturada.

De todos los blogs señalados en las respuestas, los más repetidos fueron:

\section{Nombre del blog}

Tematica

\section{Menciones}




$\begin{array}{lll}\text { Tras la pista de Paula } & \text { Moda } & 2,2 \% \\ \text { Blog de cine } & \text { Cine } & 2 \% \\ \text { Escolar.net } & \text { Politica } & 2 \% \\ \text { En la boca del lobo }{ }^{1} & \text { Politica } & 2 \% \\ 233 \text { grados } & \text { Periodismo } & 1,5 \% \\ \text { El blog de Clara Alonso } & \text { Moda } & 0,9 \% \\ \text { Futbolitis } & \text { Deportes } & 0,9 \%\end{array}$

En el caso de los dos blogs de moda más mencionados, el blog de la actriz Paula Echevarría Tras la pista de Paula alojado en Elle.es, y el blog de la modelo Clara Alonso, El blog de Clara Alonso, alojado en Vogue.es, muestran el mayor poder prescriptor de los "blogs empotrados" en medios de comunicación, unido en este caso además a una "celebrity".

Blog de cine es una publicación empotrada en Weblogs SL, un proyecto con más de 40 publicaciones propias y un tráfico conjunto, según datos de febrero de 2013, de 12,75 millones de usuarios únicos al mes.

Entre los blogs de periodismo el más mencionado, si bien con un 1,5\%, es 233 grados, del medio digital Lainformacion.com.

Escolar.net está empotrado en eldiario.es, del que Ignacio Escolar es director.

Futbolitis es el blog empotrado en Marca que escribe el colaborador del diario, entre otros medios, Marcos López.

Frente a este tipo de "blogs empotrados", las voces individuales de bloggers fuera de los medios pasan a formar parte del consumo hiper-fragmentado. Dentro de estas otras voces figuran otros ejemplos, citados por menos del $0,5 \%$ de las respuestas, como Cinéfilo y medio, o El fútbol es nuestro. El resto de los blogs anotados se citan como máximo dos o tres veces en el total de las 438 menciones.

Un dato que avala la tesis de la mayor actividad de los bloggers en la web 2.0 y el poder prescriptor de los blogs referentes de los jóvenes en la Red es que si comparamos esta repuesta sobre consumo temático de blogs, con las temáticas de los jóvenes encuestados autores de un blog $(40,1 \%)$, los resultados casi coinciden exactamente. El orden de temáticas de los jóvenes encuestados con blog sería la siguiente: política, deportes, cine y música.

\footnotetext{
1 Algunos encuestados apuntan el nombre del antiguo blog de Ramón Lobo en El Pais.com, "Aguas internacionales" pese a que dejó de publicarse en noviembre de 2012 tras su despido del diario.
} 


\section{8-25 años $\| \mathrm{N}: 500$}

TENDENCIAS DE CONSUMO DE BLOGS POR LOS JÓVENES

11b/14: EN CASO AFIRMATIVO INDICA LA TEMÁTICA

Provincia: Todas Participantes según sexo: $181 \mathrm{H} / 319 \mathrm{M}$ Edad: $18-25$ años $\quad \mathbf{N}^{\circ}$ total de paticipantes: 500

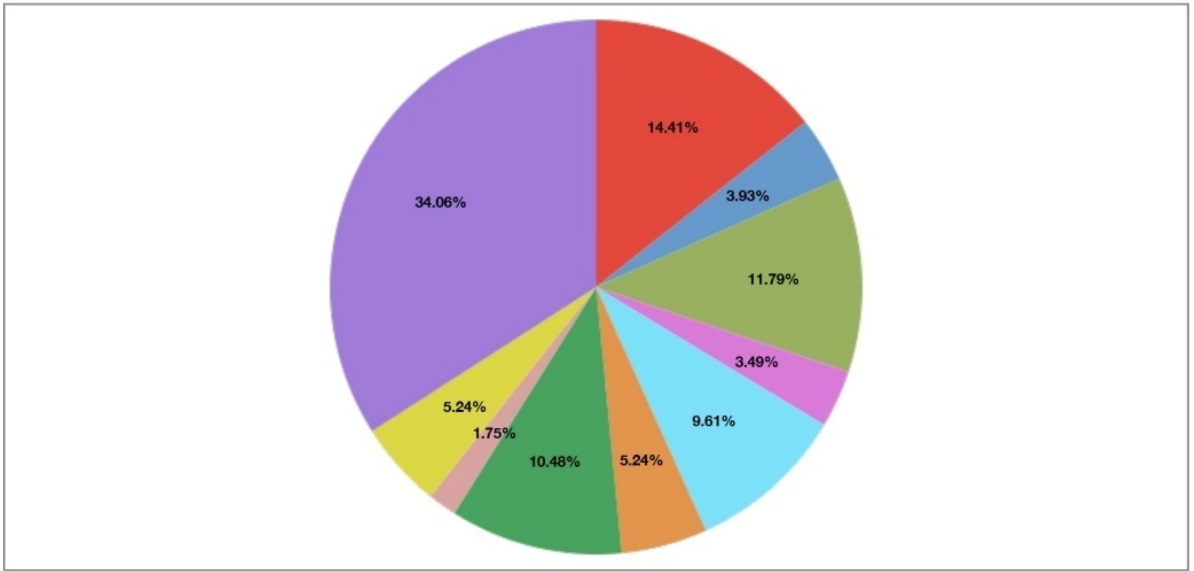

- Politica $=$ Tecnología $\mid$ Deportes $\mid$ Viajes $=$ Música $\mid$ Moda $\mid$ Cine $=$ Cocina $=$ Libros $=$ Otros

De esto se desprende, también, que los bloggers son, en el caso de la franja elegida para el estudio (18-25 años), los principales lectores y consumidores de blogs, llevando a cabo la denominada "práctica multisituada" (Estalella, 2006, p. 27), según la cual el blogger no sólo escribe en el blog, sino que lee otros blogs, los enlaza..

A la cuestión sobre si "confian y siguen las recomendaciones de los blogs" sólo el 11,3\% afirma no hacerlo nunca, -casi la misma cifra de los que aseguran hacerlo casi siempre (9,9\%) o siempre (1,8\%)- pero una mayoría reconoce seguirlas " a veces" (76,8\%).

\section{8-25 años || N: 500 \\ TENDENCIAS DE CONSUMO DE BLOGS POR LOS JÓVENES}

10/14: ¿CONFÍAS Y SIGUES LAS RECOMENDACIONES DE LOS BLOGGERS A LOS QUE SIGUES?

Provincia: Todas Participantes según sexo: $181 \mathrm{H} / 319 \mathrm{M}$ Edad: $18-25$ años $\quad \mathbf{N}^{\circ}$ total de paticipantes: 500

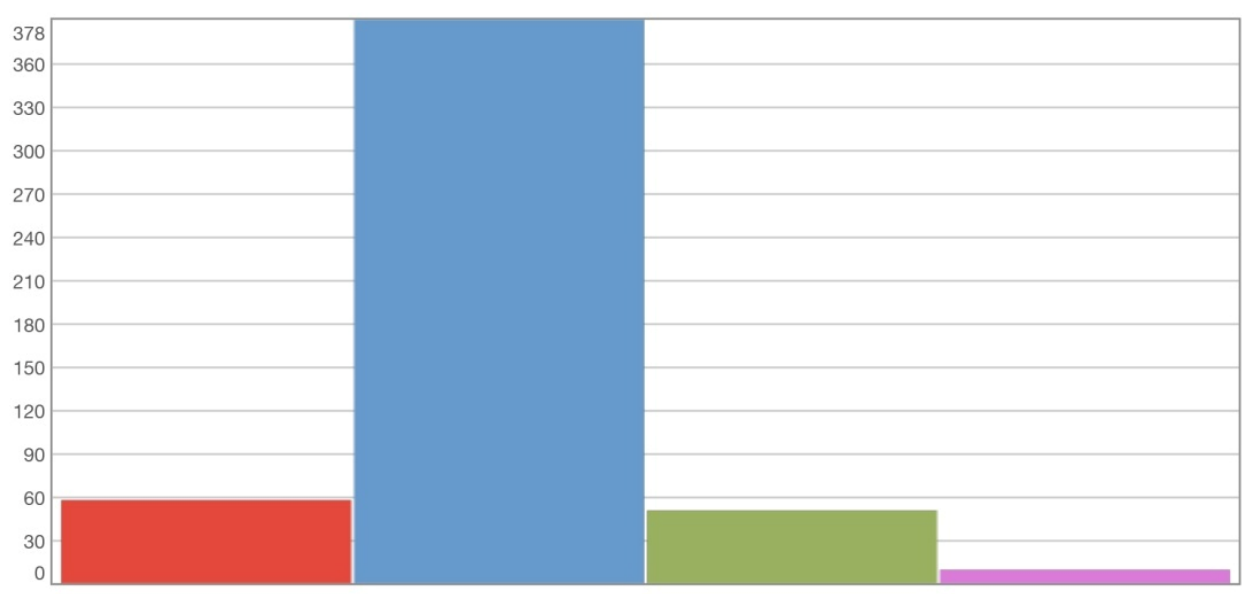

" Nunca $=$ A veces $=$ Casi siempre $\mid$ Siempre

Pese al consumo hiper-fragmentado de blogs que acabamos de describir, que muestra la dificultad de fidelizar a una audiencia que consume blogs, sí se comprueba no obstante la credibilidad que tienen los bloggers entre sus lectores.

\subsection{Participación e interactuación en los blogs que siguen}

La participación en la blogosfera por parte de los jóvenes objeto de esta investigación tanto a través de comentarios a los posts, como su envio por Twitter a otros amigos, está en relación con el propio hábito y tiempo de consumo de los mismos, así como de lo que buscan y 
les aporta la lectura de blogs.

Partiendo de los primeros resultados de este estudio (González Aldea, López Vidales y Redondo, 2013), que coincidían con el declive de la blogosfera apuntado por varios autores a partir de 2008, se concluía que los blogs no lideran el ocio digital de los jóvenes universitarios españoles. Una cuarta parte $(26,6 \%)$ de los jóvenes encuestados apenas consume blogs $(10,59 \%$ lo hace "una vez al mes", y 16,08\% "casi nunca"). Si observamos además el tiempo de lectura de blogs comprobamos cómo el 29,5\% le dedica menos de 10 minutos a la semana.

Los resultados de esta investigación en cuanto a participación e interactuación confirman también la tesis de Violi en 2008 apuntando, ante la "desmesurada" extensión del fenómeno blog, las limitaciones reales de elección y participación en la blogosfera.

La saturación del fénomeno blog empieza a ser evidente con cifras que establecen que el $15,4 \%$ de los jóvenes encuestados considera que la oferta actual de blogs es "excesiva" y sólo el 4,1\% la considera insuficiente. A esto se suma que el $59,8 \%$ de los jóvenes encuestados no tiene blog. Los jóvenes más activos en la blogosfera son los que tienen su propio blog (40,1\% en esta investigación) y utilizan las redes sociales como Twitter para promocionar su blog.

Los resultados a la cuestión sobre si retuitean los posts que leen, el $32,5 \%$ asegura no hacerlo nunca, frente al 9,2\% que lo hace "casi siempre" (7,1\%) o "siempre" (2,1\%). Una amplia mayoría el 58,1\% retuitea a veces el contenido del blog/blogs que siguen.

\section{8-25 años $\|$ N: 500}

\section{TENDENCIAS DE CONSUMO DE BLOGS POR LOS JÓVENES}

07/14: ¿RETUITEAS CONTENIDOS DE LOS BLOGS?

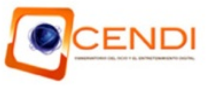

Provincia: Todas Participantes según sexo: $181 \mathrm{H} / 319 \mathrm{M}$ Edad: $18-25$ años $\quad \mathbf{N}^{\circ}$ total de paticipantes: 500

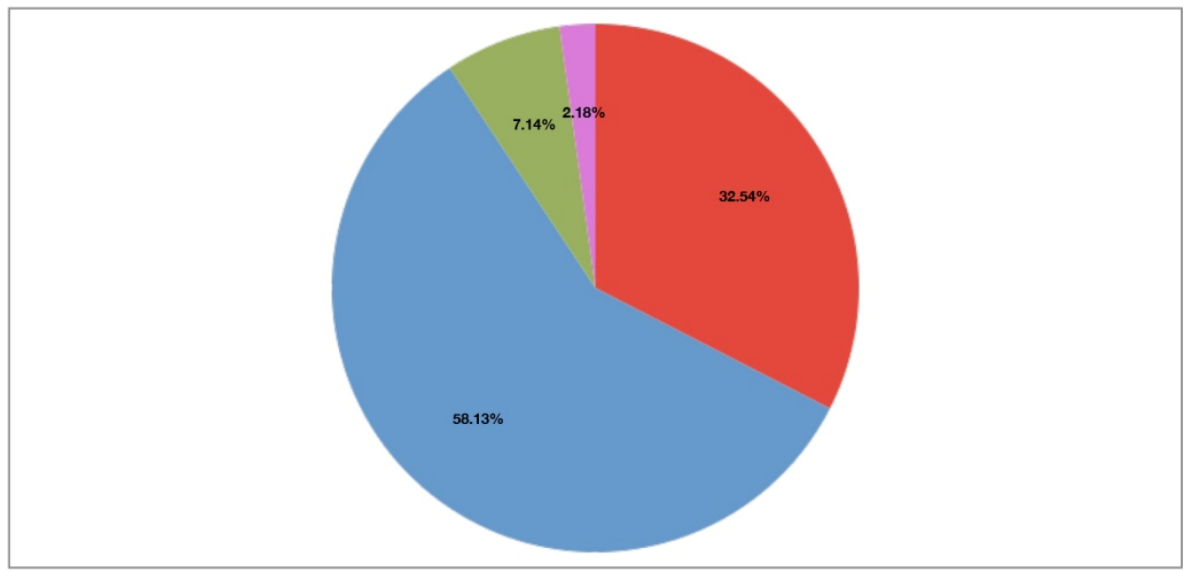

- Nunca $=$ A veces $=$ Casi siempre $=$ Siempre

El uso de Twitter es sin embargo mucho más significativo, si lo comparamos con las cifras de aquellos que escriben comentarios en los posts que leen, reflejando una participación e interactuación a través de los social media que está en consonancia con la etapa de punjanza de las redes frente al déclive de la blogosfera. 
18-25 años $\| \mathrm{N}: 500$

TENDENCIAS DE CONSUMO DE BLOGS POR LOS JÓVENES

06/14: ¿ESCRIBES COMENTARIOS EN EL BLOG QUE SIGUES SOBRE LOS POST QUE HAS LEÍDO?

Provincia: Todas Participantes según sexo: $181 \mathrm{H} / 319 \mathrm{M}$ Edad: $18-25$ años $\quad N^{\circ}$ total de paticipantes: 500

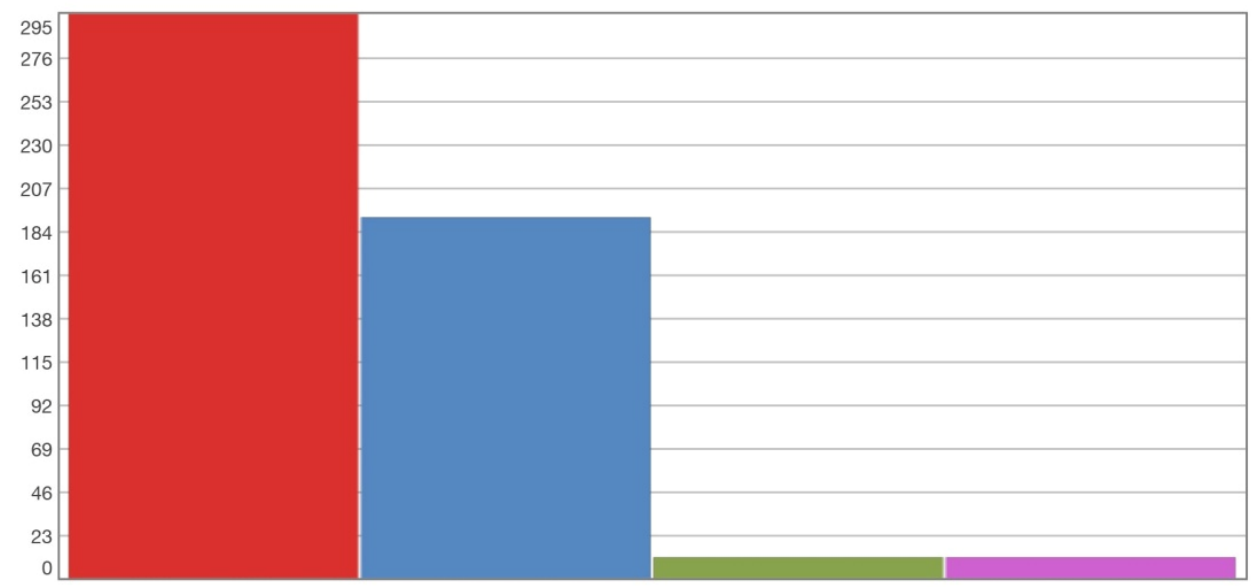

Los resultados muestran que el 58,3\% nunca escribe comentarios, (casi el doble frente al $32,5 \%$ que nunca retuitea), el $37,3 \%$ lo hace "a veces, y el 4,2\% "casi siempre" o "siempre" con un $2,1 \%$ respectivamente.

Los jóvenes buscan en la blogosfera sobre todo "información" $(38,2 \%)$ y "entretenimiento" $(31,1 \%)$. Los blogs les aportan también " orientación y consejo" (15,4\%), y la "visión personal de un experto" al 13,9\%, si bien muchos de ellos marcan varias de las opciones.

\section{8-25 años || N: 500 TENDENCIAS DE CONSUMO DE BLOGS POR LOS JÓVENES}

09/14: ¿QUÉ TE APORTA LA LECTURA DE UN BLOG?

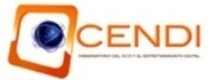

Provincia: Todas Participantes según sexo: $181 \mathrm{H} / 319 \mathrm{M}$ Edad: $18-25$ años $\quad N^{\circ}$ total de paticipantes: 500

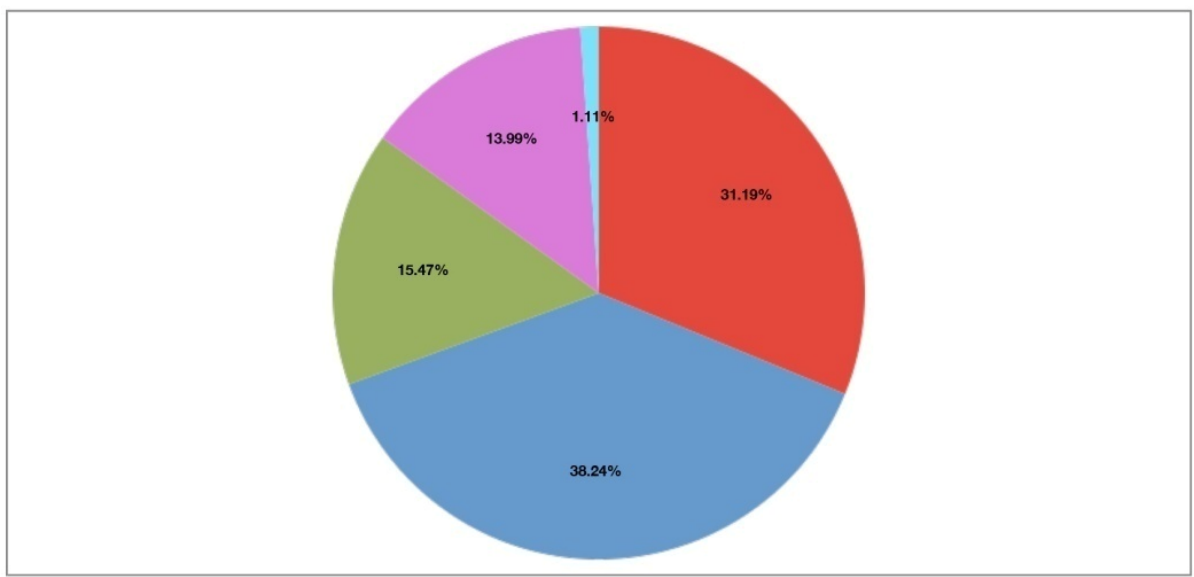

" Entretenimiento $=$ Información "O Orientación y consejo $=$ Un punto de vista más personal de un experto $=$ Otros

En principio parece más probable que sean aquellos que buscan orientación y consejo o la opinion del experto los que más interactúen a través del envio de comentarios, mientras que para compartir información y entretenimiento se utilicen más redes sociales como Twitter.

\section{Opinión de los jóvenes sobre la blogosfera}

Para conocer la opinion de los jóvenes universitarios sobre la blogosfera que de alguna forma explique los hábitos de consumo de la misma se les preguntó acerca de lo que más y lo que menos les gustaba de la misma.

Entre los aspectos más valorados de los blogs destacaron que " informan de lo último" (20,2\%), " su especialización” (19,9\%), "la opinión 
del blogger" (19,1\%) y las fotos (16,2\%), algo que se entiende si tenemos en cuenta que entre sus temáticas preferidas en los blogs están la moda, el cine o el deporte, donde estos tres parametros resultan imprescindibles.

A mucha más distancia, se sitúan en sus preferencias en los blogs cuestiones como el diseño, la frecuencia de los posts o los comentarios.

Entre lo que menos les gusta de los blogs, los jóvenes universitarios encuestados apuntan en primer lugar " que estén mal escritos, sin calidad" $(20,3 \%)$, y la publicidad $(17,4 \%)$. A más distancia sitúan "el plagio de contenidos" (11,8\%), "no citar las fuentes" $(9,2 \%)$, "la extensión excesiva de los posts" (8,9\%), "la falta de rigor" ( $8,9 \%$ ) y "los posts patrocinados" (8,8\%).

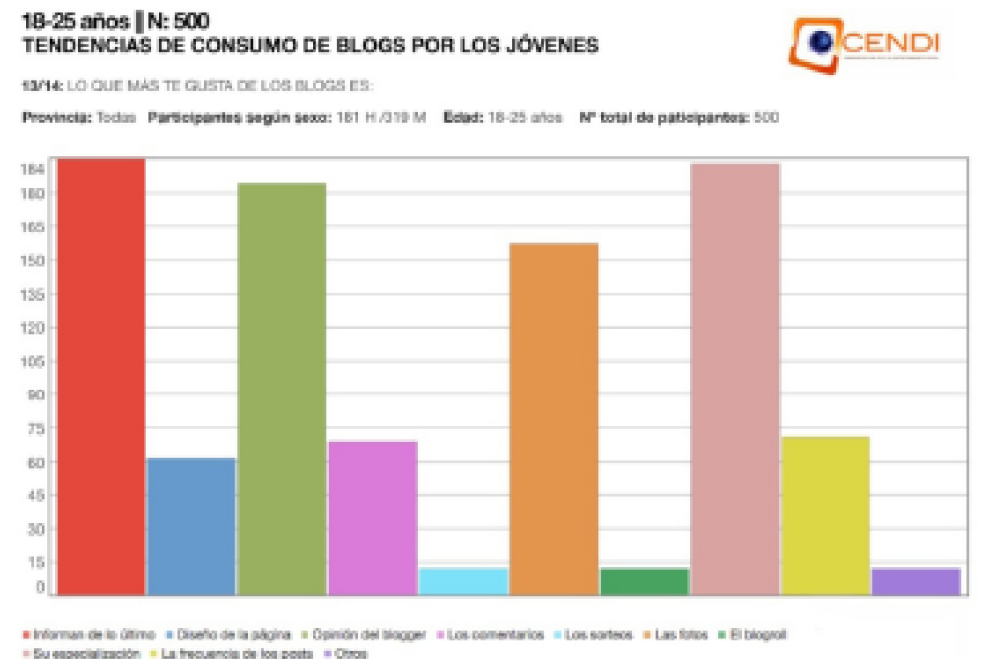

\section{Conclusiones}

El $60 \%$ de los jóvenes encuestados en esta investigación señala contar con un blog propio, siendo la política, los deportes, el cine y la música los temas preferidos que les impulsan a ello. Junto a esto, lo que más les gusta de los blogs es que "informan de lo último" y "su especialización". De hecho, un 38\% valora especialmente la información que aportan, a lo que se suma un 31\% que habla de los blogs como una herramienta entretenida.

Sin embargo, su participación en la blogosfera se limita a la lectura, ya que un significativo 32,5\% de los jóvenes reconoce que nunca retuitea contenidos entre sus amigos, frente al $58 \%$ que señala hacerlo a veces, al tiempo que seis de cada diez reconocen que nunca escriben comentarios sobre los post que leen.

La influencia y poder prescriptor de la blogosfera en estos jóvenes, -y a pesar de la credibilidad que dan a los bloggers-, parece en declive atendiendo al consumo hiper-fragmentado de blogs, escasa fidelización y a sus opiniones sobre la saturación de la blogosfera (el $15,4 \%$ considera la oferta actual "excesiva" y sólo el $4,1 \%$ la considera "insuficiente"). A esto se suma que el $59,8 \%$ de los jóvenes encuestados no tiene blog.

Las temáticas más seguidas en la blogosfera por los jóvenes del estudio son extraordinariamente variadas y la gran mayoría se refiere a información práctica y de entretenimiento: el cine $(16,9 \%)$, la política $(14,5 \%)$ la moda $(13,8 \%)$, y la música $(13,2 \%)$ pero también los viajes, la cocina, y los libros. Sin embargo, los jóvenes apenas optan por dos de las temáticas dominantes en general en la blogosfera: tecnología y blogs personales. Además, ni siquiera en los temas preferidos, los jóvenes son un público fiel a los blogs: la mayoría es incapaz de recordar los nombres de los blogs que siguen 0 , si lo hacen, recogen el nombre de forma inexacta.

Ni siquiera el mayor poder prescriptor de los "blogs empotrados" en grandes medios de comunicación, especializados en una temática determinada, consigue muchos seguidores entre los jóvenes, como se desprende de los resultados hiper-fragmentados en la mención concreta de blogs que siguen.

Se comprueba no obstante la credibilidad que tienen los bloggers entre sus lectores que confían y siguen las recomendaciones de los blogs. Sólo el 11,3\% afirma no hacerlo nunca, pero tres de cada cuatro reconoce seguir "a veces" sus recomendaciones.

\section{Referencias bibliográficas}


Antúnez, José Luis (2005): "El impacto de los gestores de contenidos (CMS) en el despegue del fenómeno blog". En Octavio Rojas et al. (eds.): Blogs. La conversación en Internet que está revolucionando medios, empresas y a ciudadanos. Madrid: Escuela Superior de Gestión Comercial y Marketing (ESIC), pp. 49-72.

Aranda, Daniel, Sánchez, Jordi, Tabernero, Carlos y Tubella, Imma (2010): "Los jóvenes del siglo XXI: prácticas comunicativas y consumo cultural". Presentado en el Congreso Internacional AE-IC Comunicación y desarrollo en la era digital. Málaga 2010. [http://www.aeic2010malaga.org/upload/ok/204.pdf, consultado el 19/11/13].

Arroyas Langa, Enrique (2006): "Los blogs: perspectivas individuales en el espacio público". Global_ Media Journal México, $\quad 3, \quad n^{0} 6, \quad$ Disponible en [http://gmje.mty.itesm.mx/arroyas.htm, consultado el 01/11/13].

Bitácoras.com (2012): "Informe sobre el estado de la blogosfera hispana 2011". Disponible en [http://bitacoras.com/informe, consultado el 06/06/13].

Ebuzzing (2013). Top Blogs-general-Noviembre2013 [http://labs.ebuzzing.es/top-blogs, consultado el 31/10/13].

Estalella, Adolfo (2006). "La construcción de la blogosfera: yo soy mi blog (y sus conexiones)".

En J. M. Cerezo: La blogosfera hispana: pioneros de la cultura digital. Madrid: Fundación France Telecom. pp. 20-38.

Estalella, Adolfo (2005): "Anatomía de los blogs. La jerarquía de lo visible". Telos, 65, octubre- diciembre. Disponible en: [http://sociedadinformacion.fundacion.telefonica.com/telos/articulocuaderno.asp@idarticulo\%3D9\&re v\%3D65.htm, consultado el 02/10/13].

Flores, Dorismilda (2010): "Miradas propias y ajenas: el sentido de la reflexividad en el blogging autobiográfico". Razón y Palabra, 73. Disponible en: [http://razonypalabra.org.mx/N/N73/MonotematicoN73/07-M73Flores.pdf, consultado el 02/11/13].

Fundación Orange (2012): El Informe eEspaña $2011 . \quad$ Disponible en: [http://fundacionorange.es/fundacionorange/analisisprospectiva.html, consultado el 01/11/13].

Garrido, Fernando y Lara, Tíscar (2008): "Perfil del blogger hispano. III Encuesta a Bloggers". Revista Diálogos de la Comunicación, nº 76.

González Aldea, López Vidales y Redondo (2013): "Tendencias de consumo de blogs entre los jóvenes universitarios españoles : radiografía de su blogosfera". Revista Historia y Comunicación Social (en publicación).

Islas, Octavio (2010): "Comprendiendo el tránsito de la blogósfera a la twittósfera". En Octavio islas y Paola Ricaurte (coords): Investigar las redes sociales. Comunicación total en la sociedad de la ubicuidad. México: Tecnológico de Monterrey, pp. 76-87. Disponible en: [http://www.razonypalabra.org.mx/Libro_IRS/InvestigarRedesSociales.pdf, consultado el 19/10/13].

Leibrandt, Isabella (2006): "El weblog, un nuevo género de la cultura popular digital". Culturas Populares, vol. 3. pp. 1-18 Disponible en: [http://www.culturaspopulares.org/textos3/articulos/leibrandt.pdf, consultado el 02/11/13].

Marta-Lazo, Carmen; Martínez-Rodrigo, Estrella y Sánchez-Martín, Lourdes (2013): "La «i- Generación» y su interacción en las redes sociales. Análisis de Coca-Cola en Tuenti". Comunicar [http://www.revistacomunicar.com/index.php?contenido=detalles\&numero=40\&articulo= 40-2013-06, consultado el 02/11/13].

Martínez Sánchez, José Manuel, (2008). El hombre posmoderno. Un enfoque humanístico. Lulu.com.

Observatorio Nacional de las Telecomunicaciones y de la Sociedad de la Información (2013): La sociedad en red 2012. Disponible en: [http://www.ontsi.red.es/ontsi/es/estudios- informes/informe-anual-la-sociedad-en-red-2012-edici\%C3\%B3n-2013, consultado el 01/11/13].

Observatorio Vasco de la Juventud (2012): Retratos de juventud. Disponible en: [http://www.gazteaukera.euskadi.net/r58657/es/contenidos/informacion/gazteen_argazkiak_bilduma/ es_5561/adjuntos/retratos15_c.pdf, consultado el 20/11/13]

Tricas, Fernando, Merelo-Guervós, Juan Julián y Ruíz, Víctor. (2006): "El tamaño de la blogosfera: medidas y herramientas". En J.M. Cerezo, (coord.) : La blogosfera hispana: pioneros de la cultura digital, Fundación France Telecom España. Disponible en: http://fundacionorange.es/areas/25_publicaciones/la_blogosfera_hispana.pd f, consultado el 19/10/13]. 
Violi, Patrizia. (2008). "Espacio público y espacio privado en la era de internet. El caso de los blogs". En CIC Cuadernos de Información y Comunicación, vol. 13, pp. 39-59.

Weblogs. [http://www.weblogssl.com/2013/03/25-trafico-de-febrero-de-2013, consultado el 28/09/13]. 